\title{
Erratum
}

\section{Erratum: Thorp et al., "Two Routes to Incidental Memory under Arousal: Dopamine and Norepinephrine"}

In the article "Two Routes to Incidental Memory under Arousal: Dopamine and Norepinephrine," by John Thorp, David Clewett, and Monika Riegel, which appeared on pages 1790-1792 of the February 26, 2020 issue, due to a production error, funder information was omitted. The following text should be added to the acknowledgments: "D.C. was supported by National Institute of Mental Health (NIMH) Grant F32 MH114536. M.R. was supported by Polish Ministry of Science and Higher Education Grant DN/MOB/199/V/ 2017 and National Science Centre Poland Grant UMO-2015/19/N/HS6/02376." The online version has been corrected.

DOI: 10.1523/JNEUROSCI.2488-20.2020 